\title{
Acceptance of Tinnitus: Validation of the Tinnitus Acceptance Questionnaire
}

Cornelia Weise, Maria Kleinstäuber, Hugo Hesser, Vendela Westin and Gerhard Andersson

\section{Linköping University Post Print}

\section{Tweet}

N.B.: When citing this work, cite the original article.

This is an electronic version of an article published in:

Cornelia Weise, Maria Kleinstäuber, Hugo Hesser, Vendela Westin and Gerhard Andersson, Acceptance of Tinnitus: Validation of the Tinnitus Acceptance Questionnaire, 2013, Cognitive Behaviour Therapy, (42), 2, 100-115.

Cognitive Behaviour Therapy is available online at informaworldTM:

http://dx.doi.org/10.1080/16506073.2013.781670

Copyright: Taylor \& Francis (Routledge): STM, Behavioural Science and Public Health Titles http://www.routledge.com/

Postprint available at: Linköping University Electronic Press

http://urn.kb.se/resolve?urn=urn:nbn:se:liu:diva-95828 
Acceptance of Tinnitus: Validation of the Tinnitus Acceptance Questionnaire

Cornelia Weise ${ }^{\text {a, b}}$, PhD, Maria Kleinstäuber ${ }^{c}$, PhD, Hugo Hesser ${ }^{\text {b }}$, MSc, Vendela

Zetterqvist Westin ${ }^{\mathrm{b}}, \mathrm{PhD}$, and Gerhard Andersson ${ }^{\mathrm{b}, \mathrm{d}}$, PhD

Author Note

a Department of Psychology, Division of Clinical Psychology and Psychotherapy, PhilippsUniversity Marburg, Marburg, Germany

b Department of Behavioural Sciences and Learning, Linnaeus Centre HEAD, Swedish Institute for Disability Research, Linköping University, Linköping, Sweden

c Department of Clinical Psychology and Psychotherapy, Johannes Gutenberg-University of Mainz, Mainz, Germany

d Department of Clinical Neuroscience, Psychiatry Section, Karolinska Institutet, Stockholm, Sweden

Number of tables: 3

Number of figures: 3

Corresponding Author: Dr. Cornelia Weise, Philipps-University Marburg, Dept. of Psychology, Division of Clinical Psychology and Psychotherapy, Gutenbergstrasse 18, D35032 Marburg, Germany; e-mail: weise@uni-marburg.de; Phone: +49 6421282 6738; Fax: +496421282 8904 (permanent address). 
Acceptance of Tinnitus: Validation of the Tinnitus Acceptance Questionnaire 


\section{Abstract}

The concept of acceptance has recently received growing attention within tinnitus research due to the fact that tinnitus acceptance is one of the major targets of psychotherapeutic treatments. Accordingly, acceptance-based treatments will most likely be increasingly offered to tinnitus patients and assessments of acceptance-related behaviours will thus be needed. The current study investigated the factorial structure of the Tinnitus Acceptance Questionnaire (TAQ) and the role of tinnitus acceptance as mediating link between sound perception (i.e., subjective loudness of tinnitus) and tinnitus distress.

In total, 424 patients with chronic tinnitus completed the TAQ and validated measures of tinnitus distress, anxiety and depression online. Confirmatory factor analysis provided support to a good fit of the data to the hypothesised bifactor model (RMSEA $=.065$; $\mathrm{CFI}=.974 ; \mathrm{TLI}=.958 ;$ SRMR $=.032)$. In addition, mediation analysis, using a nonparametric joint coefficient approach, revealed that tinnitus specific acceptance partially mediated the relation between subjective tinnitus loudness and tinnitus distress (path $a b=5.96$; 95\% CI: 4.49, 7.69). In a multiple mediator model, tinnitus acceptance had a significantly stronger indirect effect than anxiety.

The results confirm the factorial structure of the TAQ and suggest the importance of a general acceptance factor that contributes important unique variance beyond that of the firstorder factors activity engagement and tinnitus suppression. Tinnitus acceptance as measured with the TAQ is proposed to be a key construct in tinnitus research and should be further implemented into treatment concepts to reduce tinnitus distress.

Keywords: Tinnitus, Acceptance, Questionnaire Study 


\section{Introduction $^{1}$}

Tinnitus is defined as a subjective acoustic perception in the ear(s) and/or the head in the absence of any apparent external acoustic stimulation (Hallam, Rachman, \& Hinchcliffe, 1984). Whilst many different forms of sound experiences are reported, a majority of patients describe the sound as buzzing, hissing, whistling, or ringing. Chronic tinnitus is a common health problem with a prevalence of $10-18 \%$ in the adult population (Gopinath, McMahon, Rochtchina, Karpa, \& Mitchell, 2010; Hannaford, Simpson et al., 2005; Kuttila, Kuttila, Le Bell, Alanen, \& Suonpää, 2005; Nondahl, Cruickshanks et al., 2010). However, only a small proportion of tinnitus patients, i.e., about 0.5 to $3 \%$ of the adult population, develop severe distress and experience impairment in everyday life (A. Davis \& El Refaie, 2000; Gopinath, McMahon et al., 2010; Pilgramm, Rychlick et al., 1999) In these severely suffering patients tinnitus has a substantial impact on quality of life (e.g., activity limitations, or participation restrictions) (Kennedy, Wilson, \& Stephens, 2004). Moreover, distressing tinnitus is often associated with audiological dysfunctions such as hearing impairment and noise sensitivity (Dineen, Doyle, \& Bench, 1997; Martines, Bentivegna, Martines, Sciacca, \& Martinciglio, 2010), as well as with psychological problems such as depressive symptoms, anxiety, and sleep disturbances (Kuttila, Kuttila et al., 2005; Reynolds, Gardner, \& Lee, 2004).

A variety of medical and psychotherapeutic treatments that aim at reducing tinnitus distress and associated symptoms have been developed and tested (for review, see Noble,

${ }^{1}$ Abbreviations: TAQ = Tinnitus Acceptance Questionnaire; CPAQ = Chronic Pain Acceptance Questionnaire; AAQ = Acceptance and Action Questionnaire; CFA = Confirmatory Factor Analysis; CFI = Comparative Fit Index; RMSEA = Root Mean Square Error of Approximation; TLI = Tucker-Lewis Index; SRMR = standardized root mean square residual; $\mathrm{BC}=$ bias corrected 
2008). In particular, cognitive-behavioural treatment (CBT) approaches have been shown to be effective in reducing tinnitus distress (Hesser, Weise, Westin, \& Andersson, 2011). These interventions include strategies to change both dysfunctional behaviours and cognitions associated with tinnitus (e.g., Weise, Heinecke, \& Rief, 2008; Zachriat \& Kröner-Herwig, 2004). Commonly used strategies are for instance attention shift from the tinnitus to enable habituation, exposure to noises and/or to silence to reduce avoidance behaviour, or identification and modification of dysfunctional beliefs to enhance tinnitus acceptance. In recent years, newer psychotherapeutic developments, i.e., Acceptance and Commitment Therapy (ACT; Hayes, Luoma, Bond, Masuda, \& Lillis, 2006) or Mindfulness-Based Cognitive Therapy (MBCT; Segal, Williams, \& Teasdale, 2001) have been adapted to chronic tinnitus and have also been incorporated into standard CBT tinnitus treatments (Gans, 2010; Philippot, Nef, Clauw, Romree, \& Segal, 2011; Sadlier, Stephens, \& Kennedy, 2008; Zetterqvist Westin, Schulin et al., 2011). One of the main purposes of these techniques is to encourage psychological acceptance. The concept is often referred to as a process that involves a willingness to experience internal events (i.e., thoughts, feelings or physical sensations) without using strategies that aim at avoiding or controlling such sensations. This is often promoted in therapy by training people to bring full attention to the present experience in a non-evaluative way and to enact overt behavioural choices on the basis of valued goals rather than on thoughts and feelings (Hayes, Luoma et al., 2006; Marlatt \& Kristeller, 1999). Thus, acceptance-based treatments aim at helping people behave more flexibly with regards to inner experiences and consistently with their chosen values under distressing circumstances (Hayes, Luoma et al., 2006).

The motivation for incorporating acceptance-based strategies into tinnitus treatments is partly deduced from pain research, since tinnitus has many similarities with different pain disorders (Møller, 2000, 2007). Both tinnitus and pain, for example, are longstanding, continuous perceptions, characterized by unclear pathophysiology, and insufficient success of 
medical treatments (cf. Møller, 2007). Several studies in pain research have shown that acceptance is related to less pain and psychological distress as well as to an increased functional level (McCracken, 1998; McCracken \& Eccleston, 2003; Wicksell, Ahlqvist, Bring, Melin, \& Olsson, 2008). Recent studies in tinnitus research have also given preliminary support for the positive effect of acceptance on tinnitus distress (C. G. Davis \& Morgan, 2008; Hesser, Westin, Hayes, \& Andersson, 2009; Westin, Hayes, \& Andersson, 2008; Westin, Östergren, \& Andersson, 2008). Treatment trials investigating ACT- and MBCTbased treatments show beneficial effects regarding general tinnitus distress and patients' ability to deal with this distressing condition (Hesser, Gustafsson et al., 2012; Philippot, Nef et al., 2011; Zetterqvist Westin, Schulin et al., 2011). For example, Zetterqvist Westin et al. (2011) compared the effects of an ACT-treatment to that of Tinnitus Retraining Therapy (TRT) and a wait list control group. ACT had large immediate effects on tinnitus interference in comparison to wait list, and medium effects at 18-months follow up in comparison to TRT. Philippot and colleagues (2011) investigated whether MBCT or relaxation training could increase treatment effects for tinnitus patients, when delivered in addition to psycho-education. Only the mindfulness group achieved an additive decrease in negative affectivity at follow-up.

In light of the current trends in tinnitus research (Hesser, Gustafsson et al., 2012; Philippot, Nef et al., 2011; Zetterqvist Westin, Schulin et al., 2011) it is most likely that ACTand MBCT-based treatments will be increasingly offered to tinnitus patients in the nearer future. In order to investigate their efficacy, their potential additive effects as well as changes in acceptance-related behaviours (e.g., experiential avoidance, thought suppression, or acceptance of negative events), specific and well-validated measures are needed.

Questionnaires assessing acceptance-related processes have been primarily developed in pain research, for example the Chronic Pain Acceptance Questionnaire (CPAQ; McCracken, Vowles, \& Eccleston, 2004) or the Psychological Inflexibility in Pain Scale, (PIPS; Wicksell, Renofalt, Olsson, Bond, \& Melin, 2008). Based on the items of the CPAQ (McCracken, 
Vowles et al., 2004) and the Acceptance and Action Questionnaire (AAQ; Hayes, Strosahl et al., 2004) an instrument to assess acceptance also in tinnitus - the Tinnitus Acceptance Questionnaire (TAQ) - was developed and validated by Westin et al. (2008). The TAQ has been used in clinical trials (Hesser, Gustafsson et al., 2012; Westin, Hayes et al., 2008; Zetterqvist Westin, Schulin et al., 2011) conducted in Sweden. However the TAQ has not yet been translated to and replicated in other languages and/or cross-validated. The first aim of the current study was therefore to translate the TAQ into German, and to test the suggested two-factor structure by confirmatory factor analyses in a large sample of tinnitus patients.

Moreover, we were interested in investigating the role of tinnitus acceptance for the experience of tinnitus distress. To date, there is no firmly established explanation why some people experiencing tinnitus develop and maintain distress while others learn to live with the condition and do not experience distress in the long run. Previous studies showed that tinnitus characteristics themselves (e.g., objective tinnitus loudness or duration of tinnitus) do not sufficiently explain the degree of distress (Dineen, Doyle et al., 1997; Kuk, Tyler, Russell, \& Jordan, 1990). Recent studies provide preliminary support for a role of acceptance in understanding tinnitus distress. For example, in a study of Westin et al. (2008) participants with chronic tinnitus completed a mental imagery task in a sound-proof booth under three instruction conditions. Results showed that participants who were instructed to accept upcoming thoughts, feelings, or physical sensations were able to focus on the imagery task significantly longer than participants without any particular instruction. Support for the role of acceptance was also found in a longitudinal study in which the mediating role of acceptance was investigated (Westin, Hayes et al., 2008). Acceptance fully mediated the relation between tinnitus distress at baseline and depression or life quality at a 7-months follow-up.

Building on these initial findings, the second aim of the current study was to investigate if tinnitus acceptance mediates the relation between tinnitus loudness and tinnitus distress in a cross-sectional dataset. From a theoretical point of view, acceptance can only be tested as a 
mediator since it is a behaviour in relation to tinnitus, and not an outcome in itself (cf. Andersson \& Westin, 2008). Hence, we hypothesised that tinnitus acceptance partially mediates the relation between tinnitus loudness and tinnitus distress. To determine the relative magnitude of tinnitus acceptance as a mediator, we let the TAQ compete against other theoretically plausible mediators, i.e., anxiety and depression. Depression is one of the most common comorbidities in distressed, chronic tinnitus patients (Andersson, Carlbring, Kaldo, \& Ström, 2004; Zöger, Svedlund, \& Holgers, 2006), however, it is not clear if depression is caused by a distressing tinnitus or if a depressive symptomatology increases the likelihood to experience distress. In any case, depression and depressive styles of thinking might influence the relation between the tinnitus sound itself and the amount of distress. Anxiety is another commonly occurring comorbidity that might mediate the relation between tinnitus loudness and the experience of distress (Zöger, Svedlund et al., 2006). To date, only anxiety sensitivity, defined as an individual's fear of physical symptoms based on the belief that such symptoms might be harmful, has been studied in tinnitus patients. Hesser and Andersson (2009) showed that heightened anxiety sensitivity was associated with increased tinnitus distress.

\section{Method}

\section{Participants}

Participants were recruited through two ongoing intervention studies. Study 1 compared the efficacy of an Internet-based cognitive-behavioural self-help treatment (iCBT) with the participation in an online discussion forum about tinnitus related topics. Study 2 compared the efficacy of the same iCBT with a group therapy and also an online discussion group. Participants for both studies were recruited by means of articles on several websites (e.g. website of a nationwide tinnitus self-help group), articles in newspapers and magazines, a report about the study on radio/TV, or recommendations by medical doctors or 
psychotherapists. A total of 435 people who experienced tinnitus reported interest in participation in one of the studies. The enrollment took place between April and October 2010.

Participants were included in the current study if they fulfilled the following criteria: (a) had tinnitus for more than three months; (b) were at least 18 years old; (c) had had an initial otological examination of tinnitus by an Ear-Nose-Throat (ENT) specialist (d) underwent a telephone interview including an assessment of psychiatric disorders and suicidality. Participants were excluded if the tinnitus was associated with Morbus Ménière or another untreated medical disease.

\section{Procedure}

Prior to enrollment, participants were informed about the overall study design and the study procedures. Participants reported their interest in the study via a webpage and were asked to download and read the study information along with the informed consent. Participants were required to send back one signed copy of the informed consent by mail before they received a link to a set of online-questionnaires. Next, participants were contacted via telephone for an interview including questions about study inclusion criteria, tinnitus characteristics, and comorbid psychological and physical disorders. The existence of severe psychiatric disorders was investigated on the basis of the individual results from the online survey by using the corresponding International Diagnostic Checklists for ICD-10 and DSMIV (IDCL; Janca \& Hiller, 1996). Following that, participants received a link to a webpage with the study survey including the questionnaires investigated in the current study. All questionnaires were administered via the Internet. Previous studies showed that Internet and paper-pencil versions of the same questionnaires yield comparable results and that psychometric properties tend to replicate in online administration (Andersson, KaldoSandstrom, Strom, \& Stromgren, 2003; Carlbring, Brunt et al., 2007; Hedman, Ljótsson et al., 2010). The study protocol was approved by the ethics committee at the site. 
Measures

Demographic and tinnitus-associated variables

In addition to demographic data, tinnitus-associated variables were assessed, for example tinnitus duration, noise sensitivity, or hearing impairment. Furthermore, patients rated on a 5-point scale from very quiet to very loud the subjectively perceived loudness of their tinnitus during the last week.

\section{Tinnitus Acceptance Questionnaire (TAQ)}

The TAQ was developed on the basis of pain acceptance questionnaires, i.e., seven items from the CPAQ (McCracken, Vowles et al., 2004) and two from the AAQ (Hayes, Strosahl et al., 2004) were adapted for tinnitus (Westin, Hayes et al., 2008). To address further contents, Westin et al. created three further items in formal accordance with the other item's style. Thus, the tested questionnaire consisted of 12 items rated on a 7-point Likert scale from never true to always true. Eight items are reversed in scoring so that high values indicate high levels of acceptance. The first version was pilot tested and items were reworded to increase comprehensibility.

An exploratory factor analysis revealed a two-factor structure with eigenvalues of 5.66 and 1.76, respectively (Westin, Hayes et al., 2008). The two factors accounted for $61.8 \%$ of the variance in the TAQ (factor 1: 47.2\%; factor 2: 14.7\%). Nine out of 12 items loaded on the first factor named activity engagement (loadings from .51 to .81), the remaining three items loaded on the factor tinnitus suppression (loadings from .66 to .87). All 12 items were retained in the final version. The factor activity engagement is conceptually related to behavioural activation and assesses if or to what extent a person continues everyday life activities regardless of tinnitus. The factor tinnitus suppression measures attempts to control thoughts and feelings concerning tinnitus and represents therefore a measure of experiential avoidance. This is due to the fact that acceptance itself is often out of people's awareness. 
Thus, assessments often target the opposite of acceptance, i.e., experiential avoidance. Items of the tinnitus suppression subscale are recoded, i.e., low scores on the scale indicate a low level of acceptance.

Results of the reliability analysis showed an internal consistency of Cronbach's alpha of .89 for the total scale, and of $\alpha=.91$ and $\alpha=.70$ for the factor activity engagement and tinnitus suppression, respectively (Westin, Hayes et al., 2008). The correlation between the factors was $r=.30$, the correlation between the entire scale and the factors was $r=.94$ for the first, and $r=.59$ for the second factor (all $p^{\prime} \mathrm{s}<.01$ ). To assess the construct validity, the TAQ was correlated with the AAQ (Hayes, Strosahl et al., 2004) and a correlation of $r=.35$ $(p=.002)$ was shown.

In the current study, the first step in the translation procedure involved forward translation of the original version into German, independently by two native Germans who were fluent in Swedish. After translation the translators met to agree on a common pilot version and to discuss problematic items. In the second step, a native Swede, fluent in German, translated the German version back into Swedish. The back-translation was compared with the original TAQ by the first two authors of this paper. For items where the two versions did not agree, the choice of words was discussed between the translators as well as with the original developer of the TAQ (V. Zetterqvist Westin) until a final version was reconciled.

\section{Tinnitus distress}

Tinnitus distress and severity were assessed with the Tinnitus Handicap Inventory (THI; Kleinjung, Fischer et al., 2007; Newman, Jacobson, \& Spitzer, 1996) and the Mini-Tinnitus Questionnaire (Mini-TQ; Hiller \& Goebel, 2004). The THI is one of the most commonly used self-report inventories to assess global tinnitus severity on a functional, emotional, and catastrophic subscale. The 25-items are rated on a 3-point scale (yes, sometimes, no) and add 
up to a total score ranging from 0 to 100 points. The THI allows a classification of tinnitus severity as no handicap (0-16 points), mild (18-36), moderate (38-56), and severe (58-100). Several studies support the good psychometric properties, including high internal consistency (Cronbach's alpha: $\alpha=.93$; Newman, Jacobson et al., 1996), good test-retest reliability and strong convergent validity (Baguley, Humphriss, \& Hodgson, 2000; Newman, Sandridge, \& Jacobson, 1998). In the current sample, an equally high internal consistency for the THI was found (Cronbach’s $\alpha=.93)$.

Regarding the Mini-TQ (Hiller \& Goebel, 2004), a 12-item self-report scale, participants rate their common complaints relating to tinnitus on a 3-point scale (yes, sometimes, no). The items encompass the topics emotional and cognitive distress, intrusiveness of tinnitus, and sleep disturbances. The score range is 0 to 20, with higher scores indicating higher tinnitus distress. The Mini-TQ has been shown to have good internal consistency (Cronbach's alpha: $\alpha=.90)$, test-retest reliability ( $r=.89)$ and construct validity (Hiller \& Goebel, 2004). Good internal consistency (Cronbach's $\alpha=.86$ ) was obtained in the current study.

\section{Anxiety and Depression}

The Hospital Anxiety and Depression Scale (HADS; Herrmann-Lingen, Buss, \& Snaith, 2005; Zigmond \& Snaith, 1983) was developed to screen for anxiety and depression among somatic patients. The 14 items can be rated on a scale from 0 to 3 and are divided into an anxiety and a depression subscale. The scales have shown good psychometric properties in several studies. Cronbach's alpha ranges from .80 to .93 for the anxiety and from .81 to .90 for the depression scale (Herrmann, 1997). The good psychometric properties have been confirmed in tinnitus samples (Zöger, Svedlund, \& Holgers, 2004) as well as for the Internetadministration of the HADS (Andersson, Kaldo-Sandstrom et al., 2003). Also in the current 
sample, satisfactory internal consistency was obtained for the anxiety scale (Cronbach's $\alpha=.82)$ and the depression scale ( $\alpha=.89)$.

Data Analytic Approach

All statistical analyses were performed using IBM SPSS, version 19, and IBM SPSS Amos 19.

Confirmatory Factor Analysis (CFA)

Confirmatory factor analyses (CFA) within the structural equation framework were employed to test the factorial structure of the TAQ. In the first step, a general factor model with all 12 items was tested. In the second step, the adequacy of the two-factor model as suggested by the original study (Westin, Hayes et al., 2008) was investigated (see Figure 2).

Based on theoretical assumptions about the concept of acceptance and its assessment we additionally tested a bifactor model (Chen, West, \& Sousa, 2006; Schmid \& Leiman, 1957; Yung, Thissen, \& McLeod, 1999). According to Chen et al. (2006), this model is particularly applicable when a general factor and multiple domain specific factors are of focal interest. In our study, the bifactor model consists of (a) two orthogonal, first-order factors, i.e., 'activity engagement' and 'tinnitus suppression', each of which is hypothesised to account for the unique influence of the specific domain, and (b) a higher-order, general factor, i.e., 'acceptance', which is hypothesised to account for the commonality of the items. In a bifactor model, the general factor has direct effects on all manifest variables, however, not on the firstorder factors (see Figure 3). Thus, the general factor contributes unique variance beyond that of the first-order factors. The model was identified by constraining all latent variable variances to 1 (Byrne, 2010). The bifactor model was used to be able to investigate the role of activity engagement and tinnitus suppression independently from the general acceptance factor. In addition, using the bifactor model enable us to directly examine the relationship between the first-order factors and their associated items (Chen, West et al., 2006). The CFA 
models were evaluated using established goodness-of-fit indices in structural equation modelling: chi-square-test; root-mean-square-error of approximation (RMSEA); standardized root mean square residual (SRMR); comparative fit index (CFI); and the Tucker-Lewis Index (TLI). Different cut-off levels for the fit indices have been recommended. For the RMSEA, values less than .08 reflect adequate fit while values less than .06 indicate good fit; for the SRMR values less than .05 indicate good fit; for the CFI and TLI, values above .90 suggest an acceptable fit and values above .95 a close fit of the model (Byrne, 2010; Hu \& Bentler, 1999).

\section{Mediation analysis}

To further assess the role of acceptance for the experience of tinnitus distress, mediation analyses were performed. Mediation analyses explore the impact of a mediating variable (M) on a relationship between an independent (X) and a dependent variable (Y) (Baron \& Kenny, 1986; Preacher \& Hayes, 2004). In the current study, the mediating role of tinnitus acceptance (as measured by the TAQ subscales) on the relation between subjectively perceived tinnitus loudness and actual self-reported tinnitus distress (THI) was tested. As suggested by Preacher and Hayes (2008), a multiple mediator model was investigated. That is, we let tinnitus acceptance (TAQ), depression (HADS depression), and anxiety (HADS anxiety) pit against one another within a single model (see Figure 1). In the mediation analysis, those demographic and audiological control variables were included, that showed significant zeroorder correlations with the dependent variable.

-Please insert Figure 1 about here-

Mediation was tested with Hayes' INDIRECT.SPS macro for SPSS (Preacher \& Hayes, 2008) using the bootstrap approach for multiple mediator models. Bootstrapped standard errors and 95\% bias-corrected confidence intervals for the hypothesised indirect effects were calculated using bootstrap procedures with 5000 resamples from the original data (Preacher \& Hayes, 2004). 


\section{Results}

\section{Sample Characteristics and Descriptive Statistics}

Of the 435 tinnitus patients who reported interest in participation, 11 were excluded due to the following reasons: (a) Morbus Mèniére ( $n=2)$; (b) tinnitus duration of less than three months ( $n=1)$; (c) questionnaire not completed ( $n=2)$; (d) not available for telephone interview ( $n=6)$. Thus, 424 tinnitus patients fulfilled all inclusion criteria and were included in the current analysis. Of the 424 participants, 3 had missing data on one variable. Missing data were found to be missing completely at random, i.e., study variables were not significantly associated with the presence of missing values on these variables, Little's MCAR test, $\chi 2(d f=71, N=424)=47.65, p=.985$ (Little \& Rubin, 2002). In addition, possible differences between participants who had registered for Study 1 ( $n=257)$ or Study 2 ( $n=167)$ were investigated. Comparisons across demographic and clinical characteristics did not reveal any significant differences between participants culled from the two studies (all $p$ 's $>.23$ ). Thus, all 424 participants were included in the subsequent analyses.

For the complete sample, demographic data, tinnitus characteristics, and means of the variables included in the main analyses are shown in Table 1. Of the 424 included participants, 164 patients (38.7\%) responded to articles in local newspapers and national magazines, 97 patients (22.9\%) heard about the study by word-of-mouth, 102 patients (24.1\%) read about the study on the internet, 31 patients (7.3\%) received a recommendation for participation in the study by their medical doctor or psychotherapist, and 30 patients (7.1\%) heard about the study through the radio or TV. $64.6 \%$ of the participants had a high level of education, i.e., Alevels or university degree. On average, participants were 52 years old and had their tinnitus for 8 years, showing the chronic state of tinnitus. Participants showed on average a moderate tinnitus handicap on the THI $(M=37.71, S D=19.97)$ and a moderate distress on the Mini-TQ 
$(M=11.98, S D=5.29)$. Patients rated the loudness of their tinnitus in the last week on a scale of $1-5$ on average as $3.34(S D=0.74)$.

-Please insert Table 1 about here-

Psychometric properties of the items and the subscales

The mean of the TAQ was $42.90(S D=12.70)$. The total scale as well as both subscales demonstrated good or sufficient internal consistency with Cronbach's alpha $\alpha=.86$ for the entire scale, $\alpha=.91$ for the subscale activity engagement, and $\alpha=.68$ for the subscale tinnitus suppression. Item means ranged from 3.42 to 4.56 for the subscale activity engagement, and from 2.11 to 2.45 for the subscale tinnitus suppression. Item means and item-total correlations for the total scale as well as for both subscales are listed in Table 2. The subscales were weakly inter-correlated, $r=.21, p<.001$, indicating that they were both, distinguishable and related. The correlation with the TAQ total score was $r=.94(p<.001)$ for the first subscale, and $r=.53(p<.001)$ for the second subscale.

-Please insert Table 2 about here-

Confirmatory factor analysis

First, a general factor model with all 12 items was tested. None of the goodness of fit indices reached acceptable values, $\chi 2(d f=54 ; N=424)=732.05, p<.001$, CFI $=.756$, $\mathrm{TLI}=.701, \mathrm{RMSEA}=.172, \mathrm{SRMR}=.116$. Second, the two-factor solution, as suggested by the original study (Westin, Hayes et al., 2008), was tested (see Figure 2). The result of the model comparison with the chi-square-difference test revealed that the two-factor model was to be preferred compared to the general factor model, $\Delta \chi^{2}(1)=214,13, p<.001$. However, although the goodness of fit values indicated a significantly better fit of the data to the twofactor model, indices still did not reach the recommended cut- off levels for satisfactory model fit, $\chi 2(d f=53 ; N=424)=517.92, p<.001, \mathrm{CFI}=.832, \mathrm{TLI}=.791, \mathrm{RMSEA}=.144$, SRMR $=.084$. Even after adding covariances between the error terms of several items, as 
suggested by the modification indices, the RMSEA did not reach a recommended value of less than .08 (Byrne, 2010; Hu \& Bentler, 1999).

-Please insert Figure 2 about here-

Third, the model fit of the bifactor solution was tested (see Figure 3). Fit indices provided support to an acceptable fit of the data to the hypothesised model, $\chi^{2}(d f=42$; $N=424)=168.07, p<.001$, i.e., RMSEA $=.084, \mathrm{CFI}=.955, \mathrm{TLI}=.929$, and SRMR $=.035$. Model fit of the bifactor model was significantly better than that of the two-factor model, $\Delta \chi^{2}(11)=276,92, p<.001$. Modification indices showed that adding a covariance between the error terms for items 6 and 10 would significantly improve the model fit. Given that these items are clearly related in content, it was considered appropriate to allow for these error terms to co-vary. Following this modification of the model, fit indices indicated a better fit of the data to this model, $\chi 2(d f=41 ; N=424)=114.11, p<.001$, i.e., RMSEA $=.065$, $\mathrm{CFI}=.974, \mathrm{TLI}=.958, \mathrm{SRMR}=.032$. In addition, the chi-square-difference test yielded a significant improvement in model fit between the original and the modified bifactor model, $\Delta \chi 2(1)=53.96, p<.001$. The standardized loadings of all items on the first-order and general factor are shown in Figure 3. All loadings on the first-order factors were significant and nine out of 12 loadings on the general factor reached significance.

-Please insert Figure 3 about here-

Mediation of the relation between subjectively perceived tinnitus loudness and tinnitus distress

In the first step, we tested the mediating role of the two subscales of the TAQ and the subscales anxiety and depression of the HADS. Age and hearing impairment showed significant zero-order correlations ( $r=-.20$ and $r=.19, p^{\prime} s<.001$, respectively) with the dependent variable and were thus included as covariates. In support of our hypothesis, tinnitus acceptance and anxiety had a partial mediating effect on the relation between subjectively 
perceived tinnitus loudness and tinnitus distress, even after controlling for the effect of age and hearing impairment. A statistically significant total indirect effect, reflecting the difference between the total and direct effect, was found (estimate $=7.67$; bias corrected [BC] 95\% CI: 5.68, 9.82). However, the direct effect of tinnitus loudness on tinnitus distress remained significant after controlling for the mediators. The specific indirect effects of tinnitus loudness on tinnitus distress through TAQ activity engagement (estimate $=5.19$; BC 95\% CI: 3.80, 6.97) as well as through HADS anxiety were significant (estimate = 2.36; BC 95\% CI: 1.42, 3.50). In contrast, the specific indirect effects via TAQ tinnitus suppression (estimate $=0.007$; BC 95\% CI: -0.08, 0.18), and HADS depression (estimate $=0.11$; BC 95\% CI -0.54, 0.93) were not significant.

Hence, in the second step we let only the mediators TAQ activity engagement and HADS anxiety compete. Table 3 summarizes the results for this mediation analysis. The control variables age and hearing impairment had again a significant partial effect on tinnitus distress. In accordance with the results of the first analysis, a partial mediation of the relation between subjectively perceived tinnitus loudness and tinnitus distress was found while controlling for age and hearing impairment. Results showed that the total indirect effect (estimate $=7.72$; BC 95\% CI: 5.73, 9.84) and the specific indirect effects for TAQ activity engagement, and HADS anxiety, were all significant (see Table 3). In addition, the contrast between the two competing mediators was significant (estimate $=2.98$; BC 95\% CI: 1.54, 4.68), indicating that the mediation through the TAQ activity engagement was significantly larger than the effect through HADS anxiety.

-Please insert Table 3 about here-

\section{Discussion}

The aims of the current study were to investigate the factorial structure of the Tinnitus Acceptance Questionnaire on the basis of confirmative analysis in a large sample of tinnitus 
patients. In addition, we were interested in exploring the role of tinnitus acceptance for the experience of tinnitus distress.

At first, we investigated psychometric properties of the German version of the TAQ. All patterns of results, i.e., item statistics, item-total-correlations, and subscale correlations were broadly comparable to the results reported by Westin et al. (2008). In particular, internal consistency was similar in both studies (activity engagement: $\alpha=.91$ in both studies; tinnitus suppression: $\alpha=.68$ in the current study vs. $\alpha=.70$ in the original study). The TAQ mean was slightly higher in the current sample ( $M=42.90, S D=12.70$ vs. $M=40.24 . S D=12.23)$.

Since the TAQ has to date only been investigated on the basis of exploratory factor analysis (Westin, Hayes et al., 2008), one of the objectives of our study was to test the suggested two-factor solution within the framework of CFA. This comprehensive approach allowed us to both incorporate and expand on previous findings. In a first step we tested the two-factor structure as suggested by the developers of the TAQ (Westin, Hayes et al., 2008). However, the CFA did not provide evidence for a sufficient fit of the data to the hypothesised two-factor model. One possible reason for this result might be the subscale tinnitus suppression. It could be that the number of items in this subscale is too small, or that the chosen items do not cover the concept of tinnitus suppression sufficiently. In addition, conceptual difficulties regarding the assessment of experiential avoidance as a part of acceptance might be relevant. Westin et al. (2008) have already discussed the use of the suppression subscale within the TAQ and argued that an indirect way to measure acceptance, i.e., by assessing experiential avoidance, is less obtrusive for the patients and might thus be the only feasible option. The authors decided therefore to retain the subscale in the TAQ.

This approach of including both, experiential avoidance and activity engagement when assessing acceptance is in line with current opinion in pain research. That is, acceptance is understood as a broad concept, which is constituted by different aspects (e.g., pain willingness and activity engagement) and should thus be examined as a whole and not be disintegrated (cf. 
McCracken, 2010). Therefore, in a second step of the analysis, we retained the two-factor structure, but also included a higher order factor 'acceptance'. Such a 'bifactor model' assumes heterogeneity between the lower-order factors and implies that the general factor acceptance contributes unique variance beyond that of the two lower-order factors (Chen, West et al., 2006). In contrast to the results of the mere two-factor model, fit indices provided evidence for a significantly better fit of the data to the bifactor model. The significant factor loadings on the first-order factors argue for the fact that the TAQ assesses behavioural activation and experiential avoidance, respectively. In addition, the significant loadings for nine out of the 12 items on the higher-order factor suggest that the general factor explains additional unique variance.

The different results from the two CFAs are worth commenting on. First, since the general factor does not have significant loadings on all items of the subscale activity engagement it could be assumed that the general factor has a higher relevance for the subscale tinnitus suppression than for activity engagement. That is, for the items of the subscale activity engagement sufficient variance might be explained by the first-order factor whereas for the subscale tinnitus suppression more residual variance is remaining and thus is explained by a more general acceptance factor. Second, the clearly better fit of the bifactor model and thus the fact that the acceptance factor contributes important unique variance beyond that of the first-order factors might point to an overall importance of the higher-order factor acceptance. Accordingly, it might be argued that the subscales, which were indeed only weakly inter-correlated, reflect rather distinct parts of acceptance and conjoin in a general acceptance concept. This interpretation would be in line with a more comprehensive understanding of acceptance as an overarching concept, characterized by different, interacting processes (i.e., psychological flexibility, activity engagement, willingness to experience, or experiential avoidance) (Bond \& Bunce, 2003; Bond, Hayes et al., 2011; Hayes, Luoma et al., 2006). 
Our findings resemble the conceptual difficulties connected to the assessment of acceptance. When ACT was originally conceived, acceptance was basically expressed as the converse of emotional avoidance (Hayes, Wilson, Gifford, Follette, \& Strosahl, 1996). Hence, questionnaires that were developed to assess acceptance (e.g., the AAQ or the CPAQ-R; Hayes, Strosahl et al., 2004; McCracken, Vowles et al., 2004) are mainly targeting experiential avoidance. In these questionnaires, low scores of experiential avoidance are interpreted as high acceptance and vice versa. In a similar manner, the TAQ was designed. That is, it assesses acceptance rather indirectly by measuring attempts to avoid or control tinnitus (i.e., experiential avoidance) as well as a patient's ability to pursue valued life activities and meaningful goals regardless of tinnitus (i.e., activity engagement).

Despite the conceptual difficulties, the assessment of experiential avoidance appears to be important within tinnitus research, as previous studies already showed relations between experiential avoidance strategies (i.e., thought suppression, tinnitus suppression) and tinnitus distress. For example, Andersson and colleagues (2006) examined the immediate consequences of thought suppression and found that it led to a reduction in the number of thoughts on tinnitus. Thus thought suppression produced a temporary relief from the intrusiveness of tinnitus, at least in the short term. Hesser, Pereswetoff-Morath, \& Andersson (2009) also showed that tinnitus patients who were instructed to control background sounds, experienced a temporary relief from tinnitus interference in the short term. However, in the long term, the experiential avoidance instruction resulted in increased tinnitus interference and had a negative effect on patient's cognitive performance. These findings further suggest that targeting experiential avoidance should be one important psychotherapeutic goal in order to reduce tinnitus intrusiveness in the long run. Accordingly, questionnaires that aim at evaluating treatments with a particular focus on acceptance strategies should, like the TAQ, also assess possible changes in experiential avoidance. Future studies should, however, gather further validity evidence for tinnitus suppression and investigate its integration in the 
overarching acceptance concept. For example, the TAQ could be evaluated in comparison to experimental data on tinnitus suppression, or to data on experiential avoidance in chronic pain patients.

The second aim of our study was to investigate the role of tinnitus acceptance for experiencing tinnitus distress. The conducted mediation analysis revealed that the TAQ subscale activity engagement and the HADS subscale anxiety partially mediated the relationship between the subjectively perceived tinnitus loudness and tinnitus distress. This partial mediation remained significant even after including the control variables age and hearing impairment. Activity engagement was a significantly stronger mediator than anxiety. This finding suggests that behavioural activation as one part of tinnitus acceptance can be regarded as an important factor in the understanding of tinnitus distress. That is, behaviour change in the direction of acceptance might be relevant for the development and/or maintenance of tinnitus distress. However, definite conclusions regarding the role of activity engagement for tinnitus distress cannot be drawn due to the cross-sectional design of the current study. Thus, longitudinal studies should examine if an increase in acceptance related behaviours can lower the risk for the development of tinnitus distress or reduce existing tinnitus interference.

Our result is in accordance with previous results in tinnitus and pain research. For example, Westin et al. (2008) showed that the acceptance factor activity engagement partially mediated the relation between tinnitus distress at baseline and at follow up, indicating the importance of acceptance for tinnitus experience. A study by Schutte and colleagues (2009) provided evidence for a significant association between tinnitus acceptance and lower levels of tinnitus-related distress. Similarly, Zetterqvist Westin et al. (2011) showed in their study comparing ACT with Tinnitus Retraining Therapy that changes in tinnitus distress were mediated by changes in tinnitus acceptance. Experimental research indicated that acceptance behaviour could improve tinnitus patients' performance in a cognitive test (Westin, Östergren 
et al., 2008). Furthermore, clients' verbal behaviours expressing a high level of acceptance have been shown to be successful in predicting positive outcomes following treatment of tinnitus (Hesser, Westin et al., 2009).

The partial mediation effect of the HADS subscale anxiety on the relationship between tinnitus loudness and tinnitus distress is congruent with previous results. Several studies showed an association between increased anxiety scores and the experience of tinnitus distress or maladaptive illness behaviours (Rutter \& Stein, 1999; Savastano, Marioni, \& Aita, 2007; Zöger, Svedlund et al., 2006). Higher anxiety levels and increased worrying can result in a more pronounced appraisal of tinnitus as dangerous and thus an increase in the experience of tinnitus distress.

Based on the current and previous results in tinnitus and pain research, it appears to be promising to continue including strategies aiming at tinnitus acceptance into traditional CBT programs. However, a more detailed investigation of the usefulness of such strategies within controlled treatment trials in the future is necessary to estimate the role of acceptance for the management of tinnitus.

The current study has a few shortcomings that should be considered. First, the study used data of an Internet sample of chronic, moderately suffering tinnitus patients. Investigating different samples or not using the Internet for data collection might therefore change the results. However, the current sample characteristics are broadly comparable to those of previous studies. Due to the use of an online platform, one would assume, for instance, that participants are on average younger. In fact, the age mean in the current study $(M=51.9, S D=11.95)$ is similar to the age mean $(M=52.3, S D=6.7)$ reported by the metaanalysis by Hesser et al. (2011). In our sample, a lower rate of female participants (39.4\%) was found. Other tinnitus studies show an approximately equal distribution of gender (e.g., 46.9\% females in the meta-analysis by Hesser et al.), however, some trials conducted in Germany also report lower rates of female participants, i.e., 33 - 38\% (Kröner-Herwig, 
Hebing et al., 1995; Weise, Heinecke et al., 2008; Zachriat \& Kröner-Herwig, 2004). In addition, a high level of education was found. This finding is in line with other online psychotherapy studies (e.g., Ljótsson, Hedman et al., 2011). Future research should reinvestigate the TAQ in different samples, in particular in acute tinnitus sufferers, in samples with lower/higher distress, and in samples not assessed via the Internet.

Second, the role of tinnitus suppression for acceptance remains unclear. The subscale tinnitus suppression showed a lower internal consistency and a lower correlation with the total score. Problems might result from the fact that this subscale measures a very narrow concept and that too few items were included. For example, it could have been helpful to have asked about different avoidance strategies commonly used by tinnitus sufferers, or whether or not participants attempt to suppress or distract themselves from their tinnitus. To measure tinnitus acceptance in a more direct way, it would be useful to assess whether or not tinnitus sufferers actively choose to remain in contact with their tinnitus in a non-controlling way. Further conceptual studies are needed to clarify the concept of tinnitus acceptance and its constituent parts.

Third, the mediation analysis has been conducted only on a cross-sectional basis. Cause-effect statements regarding the role of acceptance for the experience of tinnitus distress are therefore not possible. Thus, it can not be concluded if behaviour change in the direction of acceptance might be a protective factor for the development of tinnitus distress or a means to reduce existing disturbance. To further investigate the role of acceptance for tinnitus distress, longitudinal mediation analyses are necessary. In concrete terms, a study design assessing not only outcome variables but also possible mediators at pre and post treatment as well as during the course of treatment is recommended (Kazdin, 2007).

In conclusion, the factorial structure of the German version of the TAQ could be validated in the current study. Results strengthen the importance of the acceptance concept for tinnitus patients. For clinical practice the findings implicate that a stronger focus on tinnitus 
acceptance might be important to increase the individual outcome. It will thus be necessary to employ acceptance questionnaires like the TAQ in the course of tinnitus treatments. Further research in the field should focus on the conceptualization of tinnitus acceptance and experiential avoidance in tinnitus patients, the mediating role of tinnitus acceptance in the longitudinal course, and should investigate if and in which way acceptance of tinnitus can be influenced by specific interventions.

\section{Acknowledgements}

The study and the preparation of this manuscript were funded in part by a grant from the Swedish Research Council (HEAD Linnaeus grant). The authors wish to thank Kristine Jasper and Isabell Conrad for assistance in data collection and Alexander Alasjö for setting up the online questionnaire system.

\section{Disclosure Statement}

The authors have declared that no conflict of interest exists. 


\section{References}

Andersson, G., Carlbring, P., Kaldo, V., \& Ström, L. (2004). Screening of psychiatric disorders via the Internet. A pilot study with tinnitus patients. Nordic Journal of Psychiatry, 58(4), 287-291. doi: 10.1080/08039480410005792

Andersson, G., Jüris, L., Classon, E., Fredrikson, M., \& Furmark, T. (2006). Consequences of suppressing thoughts about tinnitus and the effects of cognitive distraction on brain activity in tinnitus patients. Audiology and Neurotology, 11(5), 301-309.

Andersson, G., Kaldo-Sandstrom, V., Strom, L., \& Stromgren, T. (2003). Internet administration of the Hospital Anxiety and Depression Scale in a sample of tinnitus patients. Journal of Psychosomatic Research, 55(3), 259-262.

Andersson, G., \& Westin, V. (2008). Understanding tinnitus distress: Introducing the concepts of moderators and mediators. International Journal of Audiology, 47(Suppl. 2), S178- 183.

Baguley, D. M., Humphriss, R. L., \& Hodgson, C. A. (2000). Convergent validity of the tinnitus handicap inventory and the tinnitus questionnaire. Journal of Laryngology and Otology, 114(11), 840-843.

Baron, R. M., \& Kenny, D. A. (1986). The moderator-mediator variable distinction in social psychological research: conceptual, strategic, and statistical considerations. Journal of Personality and Social Psychology, 51(6), 1173-1182.

Bond, F. W., \& Bunce, D. (2003). The role of acceptance and job control in mental health, job satisfaction, and work performance. Journal of Applied Psychology, 88(6), 1057-1067. doi: 10.1037/0021-9010.88.6.1057

Bond, F. W., Hayes, S. C., Baer, R. A., Carpenter, K. M., Guenole, N., Orcutt, H. K., . . . Zettle, R. D. (2011). Preliminary Psychometric Properties of the Acceptance and Action Questionnaire-II: A Revised Measure of Psychological Inflexibility and 
Experiential Avoidance. Behavior Therapy, 42(4), 676-688. doi:

10.1016/j.beth.2011.03.007

Byrne, B. (2010). Structural Equation Modeling with AMOS. New York: Routledge.

Carlbring, P., Brunt, S., Bohman, S., Austin, D., Richards, J., Öst, L. G., \& Andersson, G. (2007). Internet vs. paper and pencil administration of questionnaires commonly used in panic/agoraphobia research. Computers in Human Behavior, 23(3), 1421-1434. doi: 10.1016/j.chb.2005.05.002

Chen, F. F., West, S. G., \& Sousa, K. H. (2006). A comparison of bifactor and second-order models of quality of life. Multivariate Behavioral Research, 41(2), 189-225. doi: 10.1207/s15327906mbr4102_5

Davis, A., \& El Refaie, A. (2000). Epidemiology of tinnitus. In R. S. Tyler (Ed.), Tinnitus Handbook (pp. 1-21). San Diego: Singular.

Davis, C. G., \& Morgan, M. S. (2008). Finding Meaning, Perceiving Growth, and Acceptance of Tinnitus. Rehabilitation Psychology, 53(2), 128-138.

Dineen, R., Doyle, J., \& Bench, J. (1997). Audiological and psychological characteristics of a group of tinnitus sufferers, prior to tinnitus management training. British Journal of Audiology, 31(1), 27-38.

Gans, J. J. (2010). Mindfulness-based tinnitus therapy is an approach with ancient roots. Hearing Journal, 63(11), 52-56. doi: 10.1097/01.HJ.0000390823.09995.f3

Gopinath, B., McMahon, C. M., Rochtchina, E., Karpa, M. J., \& Mitchell, P. (2010). Incidence, persistence, and progression of tinnitus symptoms in older adults: The blue mountains hearing study. Ear and Hearing, 31(3), 407-412.

Hallam, R. S., Rachman, S., \& Hinchcliffe, R. (1984). Psychological aspects of tinnitus. In S. Rachman (Ed.), Contributions to medical psychology (Vol. 3, pp. 31-53). Oxford: Pergamon. 
Hannaford, P. C., Simpson, J. A., Bisset, A. F., Davis, A., McKerrow, W., \& Mills, R. (2005). The prevalence of ear, nose and throat problems in the community: results from a national cross-sectional postal survey in Scotland. Family Practice, 22(3), 227-233. Hayes, S. C., Luoma, J. B., Bond, F. W., Masuda, A., \& Lillis, J. (2006). Acceptance and commitment therapy: model, processes and outcomes. [Meta-Analysis]. Behaviour Research and Therapy, 44(1), 1-25. doi: 10.1016/j.brat.2005.06.006

Hayes, S. C., Strosahl, K., Wilson, K. G., Bissett, R. T., Pistorello, J., Toarmino, D., . . . McCurry, S. M. (2004). Measuring experiential avoidance: A preliminary test of a working model. Psychological Record, 54(4), 553-578.

Hayes, S. C., Wilson, K. G., Gifford, E. V., Follette, V. M., \& Strosahl, K. (1996).

Experiential avoidance and behavioral disorders: A functional dimensional approach to diagnosis and treatment. Journal of Consulting and Clinical Psychology, 64(6), 1152-1168.

Hedman, E., Ljótsson, B., Rück, C., Furmark, T., Carlbring, P., Lindefors, N., \& Andersson, G. (2010). Internet administration of self-report measures commonly used in research on social anxiety disorder: A psychometric evaluation. Computers in Human Behavior, 26(4), 736-740. doi: 10.1016/j.chb.2010.01.010

Herrmann, C. (1997). International experiences with the Hospital Anxiety and Depression Scale--a review of validation data and clinical results. Journal of Psychosomatic Research, 42(1), 17-41.

Herrmann-Lingen, C., Buss, U., \& Snaith, R. P. (2005). HADS-D - Hospital anxiety and depression scale (German version) (Vol. 2). Bern: Huber.

Hesser, H., \& Andersson, G. (2009). The role of anxiety sensitivity and behavioral avoidance in tinnitus disability. International Journal of Audiology, 48(5), 295-299. doi: 10.1080/14992020802635325 
Hesser, H., Gustafsson, T., Lundén, C., Henrikson, O., Fattahi, K., Johnsson, E., . . . Andersson, G. (2012). A randomized controlled trial of internet-delivered cognitive behavior therapy and acceptance and commitment therapy in the treatment of tinnitus. J Consult Clin Psychol, 80(4), 649-661. doi: 2012-00872-001 [pii]

10.1037/a0027021

Hesser, H., Pereswetoff-Morath, C. E., \& Andersson, G. (2009). Consequences of controlling background sounds: The effect of experiential avoidance on tinnitus interference. Rehabilitation Psychology, Vol 54(4), 381-389.

Hesser, H., Weise, C., Westin, V. Z., \& Andersson, G. (2011). A systematic review and metaanalysis of randomized controlled trials of cognitive-behavioral therapy for tinnitus distress. Clinical Psychology Review, 31(4), 545-553. doi: 10.1016/j.cpr.2010.12.006

Hesser, H., Westin, V., Hayes, S. C., \& Andersson, G. (2009). Clients' in-session acceptance and cognitive defusion behaviors in acceptance-based treatment of tinnitus distress. Behaviour Research and Therapy, 47(6), 523-528.

Hiller, W., \& Goebel, G. (2004). Rapid assessment of tinnitus-related psychological distress using the Mini-TQ. International Journal of Audiology, 43(10), 600-604.

Hu, L. T., \& Bentler, P. M. (1999). Cutoff criteria for fit indexes in covariance structure analysis: Conventional criteria versus new alternatives. Structural Equation Modeling, 6(1), 1-55. doi: 10.1080/10705519909540118

Janca, A., \& Hiller, W. (1996). ICD-10 checklists - A tool for clinicians' use of the ICD-10 classification of mental and behavioral disorders. Comprehensive Psychiatry, 37(3), 180-187. doi: 10.1016/s0010-440x(96)90034-6

Kazdin, A. E. (2007). Mediators and mechanisms of change in psychotherapy research. Annual Review of Clinical Psychology, 3, 1-27. doi: 10.1146/annurev.clinpsy.3.022806.091432 
Kennedy, V., Wilson, C., \& Stephens, D. (2004). Quality of life and tinnitus. Audiological Medicine, 2(1), 29-40.

Kleinjung, T., Fischer, B., Langguth, B., Sand, P. G., Hajak, G., Dvorakova, J., \& Eichhammer, P. (2007). Validierung einer deutschsprachigen Version des "Tinnitus Handicap Inventory" [Validation of the German-Version Tinnitus Handicap Inventory (THI)]. Psychiatrische Praxis, 34(Suppl. 1), 140-142. doi: 10.1055/5-2006-940218

Kröner-Herwig, B., Hebing, G., van Rijn Kalkmann, U., Frenzel, A., Schilkowsky, G., \& Esser, G. (1995). The management of chronic tinnitus--comparison of a cognitivebehavioural group training with yoga. Journal of Psychosomatic Research, 39(2), 153165.

Kuk, F. K., Tyler, R. S., Russell, D., \& Jordan, H. (1990). The psychometric properties of a tinnitus handicap questionnaire. Ear and Hearing, 11(6), 434-445.

Kuttila, S., Kuttila, M., Le Bell, Y., Alanen, P., \& Suonpää, J. (2005). Recurrent tinnitus and associated ear symptoms in adults. International Journal of Audiology, 44(3), 164-170.

Little, R., \& Rubin, D. (2002). Statistical analysis with missing data (2nd ed.). New Jersey: John Wiley \& Sons, Inc.

Ljótsson, B., Hedman, E., Andersson, E., Hesser, H., Lindfors, P., Hursti, T., . . Andersson, G. (2011). Internet-Delivered Exposure-Based Treatment vs. Stress Management for Irritable Bowel Syndrome: A Randomized Trial. American Journal of Gastroenterology, 106(8), 1481-1491. doi: Doi 10.1038/Ajg.2011.139

Marlatt, G. A., \& Kristeller, J. L. (1999). Mindfulness and Meditation. In W. R. Miller (Ed.), Integrating spirituality into treatment (pp. 67-84). Washington D.C. : APA.

Martines, F., Bentivegna, D., Martines, E., Sciacca, V., \& Martinciglio, G. (2010). Assessing audiological, pathophysiological and psychological variables in tinnitus patients with or without hearing loss. European Archives of Otorhinolaryngology, 267(11), 16851693. 
McCracken, L. M. (1998). Learning to live with the pain: Acceptance of pain predicts adjustment in persons with chronic pain. Pain, 74(1), 21-27. doi: 10.1016/s03043959(97)00146-2

McCracken, L. M. (2010). Toward understanding acceptance and psychological flexibility in chronic pain. Pain, 149(3), 420-421. doi: 10.1016/j.pain.2010.02.036

McCracken, L. M., \& Eccleston, C. (2003). Coping or acceptance: What to do about chronic pain? Pain, 105(1-2), 197-204. doi: 10.1016/s0304-3959(03)00202-1

McCracken, L. M., Vowles, K. E., \& Eccleston, C. (2004). Acceptance of chronic pain: Component analysis and a revised assessment method. Pain, 107(1-2), 159-166. doi: 10.1016/j.pain.2003.10.012

Møller, A. R. (2000). Similarities between severe tinnitus and chronic pain. J Am Acad Audiol, $11,115-124$.

Møller, A. R. (2007). Tinnitus and pain. In B. Langguth, G. Hajak, T. Kleinjung, A. Cacace \& A. R. Moller (Eds.), Tinnitus: Pathophysiology and Treatment (Vol. 166, pp. 47-53). Amsterdam: Elsevier Science.

Newman, C. W., Jacobson, G. P., \& Spitzer, J. B. (1996). Development of the Tinnitus Handicap Inventory. Arch Otolaryngol Head Neck Surg, 122(2), 143-148.

Newman, C. W., Sandridge, S. A., \& Jacobson, G. P. (1998). Psychometric adequacy of the Tinnitus Handicap Inventory (THI) for evaluating treatment outcome. J Am Acad Audiol, 9(2), 153-160.

Noble, W. (2008). Treatments for tinnitus. Trends in Amplification, 12(3), 236-241.

Nondahl, D. M., Cruickshanks, K. J., Wiley, T. L., Klein, B. E. K., Klein, R., Chappell, R., \& Tweed, T. S. (2010). The ten-year incidence of tinnitus among older adults. International Journal of Audiology, 49(8), 580-585. doi: doi:10.3109/14992021003753508 
Philippot, P., Nef, F., Clauw, L., Romree, M., \& Segal, Z. (2011). A Randomized Controlled Trial of Mindfulness-Based Cognitive Therapy for Treating Tinnitus. Clinical psychology \& psychotherapy. doi: 10.1002/cpp.756

Pilgramm, M., Rychlick, R., Lebisch, H., Siedentop, H., Goebel, G., \& Kirchhoff, D. (1999). Tinnitus in the Federal Republic of Germany: A representative epidemiological study. In J. Hazell (Ed.), Proceedings of the 6th International Tinnitus Seminar, Cambridge (pp. 64-67). London: The Tinnitus and Hyperacusis Centre. .

Preacher, K. J., \& Hayes, A. F. (2004). SPSS and SAS procedures for estimating indirect effects in simple mediation models. Behavior Research Methods, Instruments, and Computers, 36(4), 717-731.

Preacher, K. J., \& Hayes, A. F. (2008). Asymptotic and resampling strategies for assessing and comparing indirect effects in multiple mediator models. Behavior Research Methods, 40(3), 879-891. doi: 10.3758/brm.40.3.879

Reynolds, P., Gardner, D., \& Lee, R. (2004). Tinnitus and psychological morbidity: a crosssectional study to investigate psychological morbidity in tinnitus patients and its relationship with severity of symptoms and illness perceptions. Clinical Otolaryngology, 29(6), 628-634.

Rutter, D. R., \& Stein, M. J. (1999). Psychological aspects of tinnitus: A comparison with hearing loss and ear, nose and throat disorders. Psychology and Health, 14(4), 711718.

Sadlier, M., Stephens, S. D. G., \& Kennedy, V. (2008). Tinnitus rehabilitation: A mindfulness meditation cognitive behavioural therapy approach. Journal of Laryngology and Otology, 122(1), 31-37.

Savastano, M., Marioni, G., \& Aita, M. (2007). Psychological characteristics of patients with Ménère's disease compared with patients with vertigo, tinnitus, or hearing loss. Ear, Nose and Throat Journal, 86(3), 148-156. 
Schmid, J., \& Leiman, J. M. (1957). The development of hierarchical factor solutions. Psychometrika, 22(1), 53-61. doi: 10.1007/bf02289209

Schutte, N. S., Noble, W., Malouff, J. M., \& Bhullar, N. (2009). Evaluation of a model of distress related to tinnitus. International Journal of Audiology, 48(7), 428-432. doi: $10.1080 / 14992020802716786$

Segal, Z. V., Williams, J. M. G., \& Teasdale, J. D. (2001). Mindfulness-Based Cognitive Therapy for Depression: A New Approach to Preventing Relapse. New York: The Guilford Press.

Weise, C., Heinecke, K., \& Rief, W. (2008). Biofeedback-based behavioral treatment for chronic tinnitus: Results of a randomized controlled trial. Journal of Consulting and Clinical Psychology, 76(6), 1046-1057. doi: 10.1037/a0013811

Westin, V., Hayes, S. C., \& Andersson, G. (2008). Is it the sound or your relationship to it? The role of acceptance in predicting tinnitus impact. Behaviour Research and Therapy, 46(12), 1259-1265.

Westin, V., Östergren, R., \& Andersson, G. (2008). The effects of acceptance versus thought suppression for dealing with the intrusiveness of tinnitus. International Journal of Audiology, 47(Suppl. 2), 184-190.

Wicksell, R. K., Ahlqvist, J., Bring, A., Melin, L., \& Olsson, G. L. (2008). Can exposure and acceptance strategies improve functioning and life satisfaction in people with chronic pain and whiplash-associated disorders (WAD)? A randomized controlled trial. Cognitive Behaviour Therapy, 37(3), 169-182. doi: 10.1080/16506070802078970

Wicksell, R. K., Renofalt, J., Olsson, G. L., Bond, F. W., \& Melin, L. (2008). Avoidance and cognitive fusion--central components in pain related disability? Development and preliminary validation of the Psychological Inflexibility in Pain Scale (PIPS). European Journal of Pain, 12(4), 491-500. doi: 10.1016/j.ejpain.2007.08.003 
Yung, Y. F., Thissen, D., \& McLeod, L. D. (1999). On the relationship between the higherorder factor model and the hierarchical factor model. Psychometrika, 64(2), 113-128.

Zachriat, C., \& Kröner-Herwig, B. (2004). Treating chronic tinnitus: Comparison of cognitive-behavioural and habituation-based treatments. Cognitive Behaviour Therapy, 33(4), 187-198.

Zetterqvist Westin, V., Schulin, M., Hesser, H., Karlsson, M., Noe, R. Z., Olofsson, U., .. . Andersson, G. (2011). Acceptance and Commitment Therapy versus Tinnitus Retraining Therapy in the treatment of tinnitus: A randomised controlled trial. Behaviour Research and Therapy, 49(11), 737-747. doi: 10.1016/j.brat.2011.08.001 Zigmond, A. S., \& Snaith, R. P. (1983). The hospital anxiety and depression scale. Acta Psychiatrica Scandinavica, 67(6), 361-370.

Zöger, S., Svedlund, J., \& Holgers, K. M. (2004). The Hospital Anxiety and Depression Scale (HADS) as a screening instrument in tinnitus evaluation. International Journal of Audiology, 43(8), 458-464.

Zöger, S., Svedlund, J., \& Holgers, K. M. (2006). Relationship between tinnitus severity and psychiatric disorders. Psychosomatics, 47(4), 282-288. doi: 10.1176/appi.psy.47.4.282 